\title{
Caching Strategies in Opportunistic Networks
}

\author{
Sylvia T. Kouyoumdjieva, Sanpetch Chupisanyarote, Ólafur R. Helgason, and Gunnar Karlsson \\ KTH School of Electrical Engineering and \\ Linneaus Center ACCESS \\ Stockholm, Sweden \\ \{stkou, sanpetch, olafurr, gk\} akth.se
}

\begin{abstract}
In this paper we examine content distribution in opportunistic networks. We design and evaluate strategies by which a node fetches and shares contents on behalf of other nodes, even when the contents are not of its own interest. We propose four relaying/caching options for improving the use of network resources: relay request on demand, hop-limit, greedy relay request, and weighted relay request with allocated caching positions. We also compare our strategies with a strategy from the literature. The proposed strategies are implemented in the OMNeT++ simulator, and evaluated on mobility traces with different churn levels. The results show that the performance of opportunistic caching is strongly dependent on the level of churn in the network. However, we find a strategy that outperforms the rest in all of the explored network settings.

Index Terms-caching strategies, opportunistic networks, content distribution
\end{abstract}

\section{INTRODUCTION}

Opportunistic wireless networks utilize the availability of individual devices for establishing communication [1] to exchange data. Protocols for opportunistic content distribution are commonly based on the publish/subscribe paradigm. A node that seeks contents is called a subscriber, and a node that makes contents available is referred to as a publisher. Thus, the willingness of nodes to share contents with others in their vicinity is essential for the content distribution. However, if nodes are willing to exchange only data in which they are interested, data dissemination becomes heavily dependent on the content popularity, as well as the mixing (e.g. mobility patterns) of nodes in the network. Therefore, we here allow nodes also to store and exchange data to which they are not subscribed: We call such content items public data. The goal is to study whether potential altruistic nodes behavior can result in increased overall system performance. In order to support this, we introduce two types of caching: private and public, as well as different relaying strategies to help nodes reach contents that are located outside of their direct communication range. We evaluate the performance of our system in terms of private data dissemination versus the overhead the system experiences due to public caching. Our study incorporates (1) development of an extensive framework for simulating a number of relaying and caching strategies in opportunistic context, (2) adoption of mobility models with different levels of churn and realism, and (3) presentation of a novel approach for relaying contents in opportunistic context such that we minimize the overhead of public contents while we favor the exchange of subscribed data.
The rest of the paper is structured as follows. We review related work in Section II. The caching strategies are outlined in Section III. The performance evaluation is described in Section IV. Results and evaluation are presented in Section V. Section VI summarizes the study, discusses our finding, and concludes the paper.

\section{RELATED WORK}

Caching strategies for opportunistic content dissemination usually make use of community formations and exploit the social roles of nodes inside such communities. The term community does not refer only to a physical place or location, neither does it encompass only people who are familiar with one another. What also unites nodes belonging to a community are their common interests.

The authors in [2] use the concept to form an overlay and implement publish/subscribe communication in delay tolerant networks. The overlay formation, which is a set of logical links, relies on community detection algorithms. In [3], nodes evaluate the most appropriate peer from which to fetch given content items; this evaluation is performed by maximizing a utility function defined by the size of the content, the probability that the content is available and the probability that the node can get access to that content. In [4], the authors present an idea of pre-fetching. A node determines whether to store contents in its cache based on analytic hierarchy process and Grey relational analysis. The authors in [5] propose three content selection strategies-uniform, most popular, and optimized social welfare-based on which nodes select to carry contents that are not of their own interest. We give further details on the optimized social welfare strategy in Section V. SocialCast [6] presents a routing protocol supporting publish/subscribe communication, which relies on prediction of node popularity for taking caching decisions. Nodes that have higher importance in the community are preferred as content carriers. The authors in [7] propose an algorithm called BUBBLE, which tries to select popular nodes (with high centrality) as relays by making a decision based on the knowledge of community structure. However, it depends on the routing protocol rather than a caching mechanism. Last the authors in [8] suggest a probabilistic model for a class of twohop relaying strategies supporting multicasting of information streams. The caching decisions in the relay nodes are made based on the feedback these nodes obtain when they deliver contents to the targeted destination. 


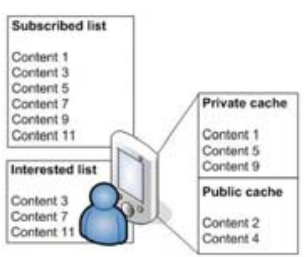

(a)

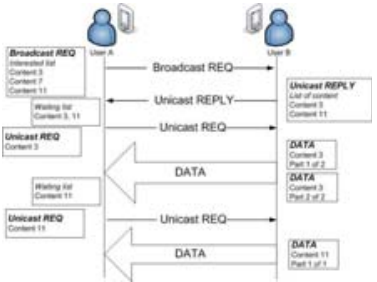

(b)
Fig. 1. (a) Example of the caching model. (b) The relay request protocol.

Our work differentiates from the ones listed above since it does not rely on the notion of community, neither does it exploit any of the presented community features. The strategies we present do not require a routing algorithm, overlay forming, or prediction of content selection. We do not utilize multicasting, as well as we do not rely on feedback to account for future caching decisions. Instead, we cache content items solely based on nodes requests.

\section{CAChing Strategies}

\section{A. Private vs. public caching}

Initially nodes subscribe only to the contents that they are privately interested in. Nodes download data from other peers only when they find content items that satisfy their private interest. We refer to this as private caching. However, since individual users have different interests, their subscriptions may vary. Therefore, the exchange of private contents depends heavily on the popularity distribution of data items stored in users' mobile devices. Nodes subscribed to highly popular contents will have more chances to obtain the data they are looking for, while nodes with interest in more specialized areas might not find peers that can serve them with the desired data. In order to address this, we propose a number of public caching strategies. Public caching allows nodes to be more altruistic and fetch contents on behalf of their peers when they cannot serve incoming requests.

In order to support public caching, each node keeps track of the available items both in its private and its public cache in an available list. Moreover, each node maintains an interested list that holds information about sought items for both caches, and a subscribed list that consists of all its private subscriptions. An illustration of the caching model is shown in Figure 1(a). A waiting list is populated with the IDs of the content items to be downloaded from a peer during a contact. We call a neighbor any node in direct communication range, and we refer to those peers which are one or more hops away from us as indirect neighbors.

\section{B. Relay Request}

The communication protocol between two neighboring nodes is illustrated in Figure 1(b). Each node periodically broadcasts Broadcast REQ messages containing the interested list. Upon reception of a broadcast request, each neighboring node checks whether it has any items in its available list that match the request. If the neighboring node cannot provide any contents, it discards the broadcast request. However, if it can partially or fully serve the request, the node sends back the IDs of the data it can provide in a unicast message destined to the peer that broadcasted the request. This peer, in turn, adds those IDs to its waiting list, to be downloaded from the peering node one content item at a time. We choose to download content items consecutively rather than in parallel because of the mobility of nodes and the relatively short contact durations.

Note that this protocol allows only for downloads from direct neighbors. However, we can virtually extend the communication range of a subscriber by having its request relayed to indirect neighbors. We here present four relay-request policies that will be combined into strategies.

1) Relay on Demand: In a dense network with high mobility and short contact durations, altruistic nodes may waste storage, transmission capacity and energy to download contents which might never be delivered to the initial requester. The relay on demand policy thus broadcasts each incoming request that cannot be served only once. If other nodes in communication range can provide the missing public content, it will be fetched and stored by the neighbor node and transferred to the actual requester upon arrival of the next broadcast request message. The neighbor node will not initiate new request attempts on behalf of the initial requester if there are no further incoming broadcast messages searching for the same content item.

2) Hop-limited Relay Request: Multi-hop relaying allows contents to be downloaded from nodes that are many hops away. However, in a network with high mobility, multi-hop relaying is not suitable. Therefore, we have a policy for relaying with a limit of two or three hops from the initial requesting node.

3) Greedy Relay Request: The previous policies assume that a node can download contents only from one neighbor at a time, no matter if this neighbor is providing contents of private or public interest. The greedy relay request relaxes this assumption by allowing nodes to constantly monitor content announcements from neighbors while at the same time downloading data from a peer. To do this, the node continues to periodically send Broadcast $R E Q$ with its interested list while associated with a peer in a download session. Whenever a new Unicast REPLY is received from a neighbor, the node checks both its current download (if it is public content) and the advertised content from the new neighbor (if it is of private interest). If both conditions are true, the node will initiate a download session to the new neighbor, and abort the current download.

4) Weighted Relay Request: Since interested lists are broadcasted, we aim to fit them into a single Broadcast $R E Q$ message. However, in dense environments due to the different private interests of peers, the number of publicly requested items that each node announces in its broadcast messages may increase significantly. When nodes are mobile, we want to be efficient when it comes to broadcasting information. Hence, we introduce the weighted relay request policy. Before sending out its interested list, a node first weighs all the content items it has been asked to provide by its neighbors since 


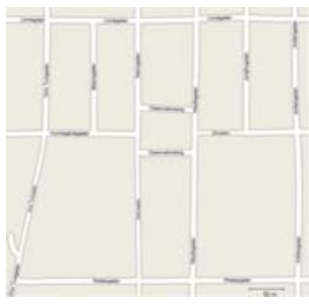

(a)

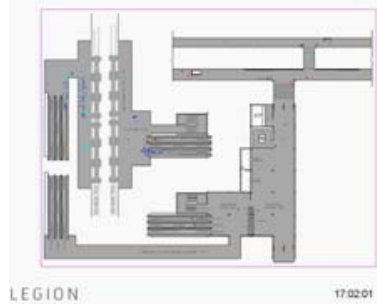

(b)
Fig. 2. High churn simulation scenarios: a part of downtown Stockholm (a) and a two-level subway station (b).

its last beacon. We account only for the requests in a single beaconing period, since we want to have quick response to the dynamics of the environment (e.g. by the time a node relays a particular request, the originator of the request may have already obtained the content item from other neighbors). The weighting process is based solely on the number of different nodes that have requested a particular item. It allocates a certain predefined number of positions $m$ for public requests in its beacon and divides them evenly among the most and the least requested content IDs. Relaying a most requested item allows peers to contribute simultaneously to a number of requesting nodes; relaying a least requested item prevents penalizing nodes with less popular interests. If any of those categories exceeds $m / 2$, the node chooses uniformly at random which IDs to include to the interested list that is broadcasted. In this work we experiment with $m=\{2,4,6\}$ allocated positions for public requests.

\section{Evaluation Scenarios}

We explore the proposed caching strategies in an opportunistic content distribution context. The mobile devices are carried by humans walking through an area. Since we believe that an opportunistic system may be viable in an urban setting, our mobility models try to reflect this.

\section{A. Modeling mobility}

Our assumption about mobility is that people tend to be in one of the following two states: either (1) on-the-go (e.g. going to the train station), or (2) on-hold (e.g. while waiting for a train to come, or while traveling in a train). Those two states differ significantly in their nature: on-the-go scenarios, on the one hand, are characterized with short contact durations with neighbors, as well as a dynamically changing set of neighbors (high churn) over time. The on-hold scenarios, on the other hand, are described by longer contact durations and a relatively constant set of neighbors (low churn) over longer periods of time. We look into each of these two categories separately.

1) High churn scenarios: For capturing human mobility in a realistic manner we use Legion Studio ${ }^{1}$, a commercial software tool for simulating pedestrian mobility in large spaces. Legion Studio allows us to study interaction of mobile nodes, such as platooning and queuing, and it permits the use of open systems, where entities can enter and leave the

\footnotetext{
${ }^{1}$ http://www.legion.com/
}

system according to predefined patterns. Each simulation run conducted in Legion Studio results in a mobility trace file, containing a snapshot of the positions of all nodes in the system every $0.6 \mathrm{~s}$.

Using Legion Studio, we explore two high churn scenarios with different levels of mobility: an outdoor urban scenario, modeling the Östermalm area of central Stockholm, Fig. 2(a), and an indoor scenario, modeling a two-level subway station, Fig. 2(b). In order to be able to compare the scenarios, we choose the input parameters such that we obtain the same average node density of 0.09 nodes $/ \mathrm{m}^{2}$ for both scenarios [9].

The outdoor Östermalm scenario consists of a grid of interconnected streets. The lengths vary between $20 \mathrm{~m}$ and $200 \mathrm{~m}$, and the width is $2 \mathrm{~m}$ to represent sidewalks. We assume that the arrival rates at all inlets to the area are equal and when nodes arrive at an intersection, they will go straight on the same street with probability 0.5 , or turn to other adjoining streets with equal probabilities. The scenarios can be characterized as a high-mobility setting since nodes are constantly on the move. The active area is $5872 \mathrm{~m}^{2}$. The communication range is assumed to be $20 \mathrm{~m}$.

The Subway scenario depicts a two-level indoor subway station. Passengers enter or leave the station through any of the entrance points on the top floor, or by trains at the platforms. The scenario is characterized by a medium level of mobility, since nodes may take breaks waiting in the coffee shop and at the train platforms. The active area of the station is $1921 \mathrm{~m}^{2}$. Due to the smaller area, we here shrink the communication range to $10 \mathrm{~m}$.

2) Low churn scenarios: The Train scenario defines a subway train with five cars; passengers enter the cars upon arrival and keep their place in the train for the rest of their journey. Passengers choose their place in the cars uniformly at random. To add realism to such a scenario, the times between stations are mapped to an actual commuter train schedule operating between the central and the northern parts of Stockholm. We assume that the number of nodes entering the train at each station is drawn from a Poisson distribution, and each nodes picks uniformly at random the end point of its journey. The length of each car is $20 \mathrm{~m}$. Nodes in a single car can efficiently communicate with each other, while communication with nodes from neighboring cars can be achieved only through relaying.

\section{B. Content initialization}

In our evaluation scenarios we assume that all nodes carry devices and that there are 1000 available content items in the area. The popularity distribution of those 1000 content items follows a Zipf distribution with parameter $\alpha=0.368$. Every device is subscribed to 10 content items upon entering the observed area, and its private cache is initially populated with 5 randomly chosen content items out of these subscriptions. Defining the way in which the subscriptions are obtained is out of the scope of the current work, however one can think that they are managed through the network in a centralized or decentralized manner. The public cache is empty upon arrival. 
TABLE I

NOTATIONS FOR DIFFERENT RELAYING STRATEGIES

\begin{tabular}{|l|l|}
\hline Notation & Relay Strategy \\
\hline \hline 1 & No caching \\
\hline $2 \mathrm{a}$ & Hop-limited relaying, 2 hops \\
$2 \mathrm{~b}$ & Hop-limited relaying, 3 hops \\
\hline $3 \mathrm{a}$ & Greedy relay request with 2 hops limit \\
$3 \mathrm{~b}$ & Greedy relay request with 3 hops limit \\
\hline $4 \mathrm{a}$ & Weighted relay request with $\mathrm{m}=2$ allocated positions \\
$4 \mathrm{~b}$ & Weighted relay request with $\mathrm{m}=4$ allocated positions \\
$4 \mathrm{c}$ & Weighted relay request with $\mathrm{m}=6$ allocated positions \\
\hline
\end{tabular}

Thus, throughout its lifetime in the simulation, each node strives to obtain the rest of the content items that belong to its subscription. Entries have a mean size of $3 \mathrm{~KB}$, and a standard deviation of $1 \mathrm{~KB}$.

\section{Simulation model}

To conduct our study, we use the $\mathrm{MiXiM}^{2}$ framework for mobile wireless networks together with the OMNeT++ simulator $^{3}$. MiXiM includes models of protocols, radio propagation, node components, and message delivery and it provides a well-constructed API for application to be run on OMNeT++. In this work we use an extension of MiXiM that supports opportunistic networking [10]. The simulation model contains the full system. Since the performance of the system depends heavily on the successful transmission and reception of Broadcast REQ messages, these messages are sent out with a periodicity of $1 \mathrm{sec}$. However, in order to avoid collisions among broadcast requests, nodes entering the system wait a random delay (between 0 and $1 \mathrm{sec}$ ) before they first broadcast a request. We believe this is a valid assumption, since in opportunistic networks nodes are by nature not synchronized.

\section{REsults}

In this section, we present the results from the simulated scenarios. We compare the results in terms of goodput, which we define as follows:

1) Private goodput - the amount of bytes downloaded in the private cache of a node, divided by the lifetime of that node in the system.

2) Public goodput - the amount of bytes downloaded in the public cache of a node, divided by the lifetime of that node in the system.

For our evaluation we use the mean values of the two goodput metrics, $\overline{G_{p r i}}$ and $\overline{G_{p u b}}$ respectively. All values of $\overline{G_{p r i}}$ are normalized with respect to the value of the No Caching strategy.

In order to develop a good understanding of the advantages and drawbacks of public caching, we examine the strategies presented in Table I. The notations in Table I are further used to refer to the strategies in the figures. Furthermore, we compare these relaying strategies with the optimal channel

\footnotetext{
${ }^{2} \mathrm{http}: / /$ mixim.sourceforge.net

${ }^{3} \mathrm{http}$ //www.omnetpp.org/
}

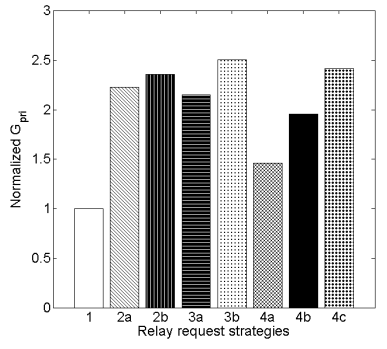

(a)

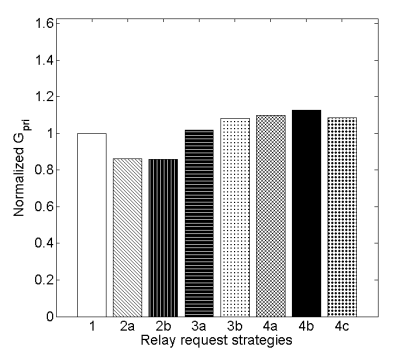

(c)

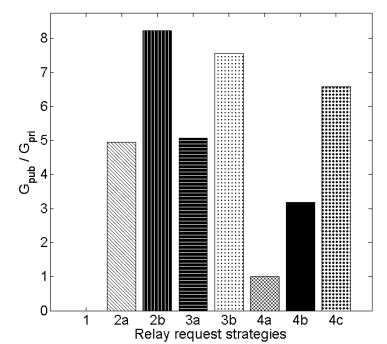

(b)

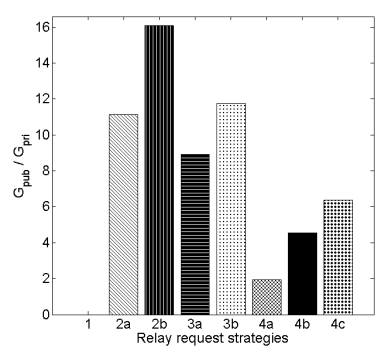

(d)
Fig. 3. Comparison of relay request strategies in high churn environments: normalized $\overline{G_{p r i}}$ and $\overline{G_{p u b}} / \overline{G_{p r i}}$ for (a, b) the Östermalm scenario and (c, d) the Subway scenario

choice strategy proposed in [5]. This strategy is based on selecting the public contents to be downloaded, as described in algorithm 2 of [5]. Each node picks a content item from its own cache (content $i$ ), as well as a content item offered by a peer (content $j$ ), and computes a probability $q$. If $\min (1, q)>$ Uniform $[0,1]$, the node will fetch and store the new item $j$ and drop its item $i$. We evaluate this strategy with different sizes of the public cache. Moreover, we present a modified version of the probability $q$ to correspond to the Zipf distribution we work with:

$$
q=\frac{\text { total numbers of nodes that store content } \mathrm{i}}{\text { total numbers of nodes that store content } \mathrm{j}}
$$

\section{A. Caching in high churn environments}

Fig. 3 shows the caching results for the normalized private goodput values and the ratio $\overline{G_{p u b}} / \overline{G_{p r i}}$ for the two high churn scenarios and the different relaying strategies. We see that both in the case of the Östermalm, Fig. 3(a), and of the Subway scenario, Fig. 3(c), the introduction of any public caching generally increases the amount of private data received by the nodes. However, when nodes are completely altruistic (strategies $2 \mathrm{a}$ and $2 \mathrm{~b}$ ), the increase in private contents comes at a price of a high overhead. The 3-hop limit in the relaying (strategy 2b) extends the communication range and increases the private goodput compared to the 2-hop case. However, it comes at a price of even higher overhead, as shown in Fig. 3(b) and 3(d). The introduction of the greedy relay request strategies ( $3 \mathrm{a}$ and $3 \mathrm{~b}$ ) partially minimizes the overhead, while at the same time delivering an equivalent user experience.

In the Östermalm scenario, Fig. 3(a), we see that when we request only 2 public items (one from the most popular, and 
TABLE II

AVERAGE SOJOURN TIME FOR DIFFERENT SCENARIOS

\begin{tabular}{|ll||ll|}
\hline \multicolumn{2}{|l||}{ High Churn } & Low Churn \\
\hline \hline Östermalm & $292 \mathrm{sec}$ & Train (normal) & $610 \mathrm{sec}$ \\
\hline Subway & $195 \mathrm{sec}$ & Train (extended) & $3260 \mathrm{sec}$ \\
\hline
\end{tabular}

one from the least popular) we achieve almost 1.5 times the private goodput we have when we do not use public caching, and this increase comes at a modest price: The amount of public content downloaded does not exceed the amount of private content. However, increasing the number of allocated positions for public contents results in higher overhead. Hence, there is an important trade-off when designing caching/relaying strategies for opportunistic networks: We want to get as much private data as possible while at the same time reducing to a minimum the public contents that have been exchanged. Such trade-off may have big impact on the user experience, when energy consumption comes into play. Mobile users would like to obtain as much contents of private interest as possible, while at the same time prolonging their battery life.

The Subway scenario shows that in such environments with very high churn level (smallest sojourn time, as shown in Table II) and high mobility, the weighted relay request with $m=2$ allocated positions does not perform well. In fact, the increase in private goodput is almost negligible, Fig. 3(c). Moreover, the hop-limited relay strategies ( $2 \mathrm{a}$ and $2 \mathrm{~b}$ ) perform worse than the No caching strategy.

We compare our strategies to the optimal channel choice strategy, applied to the Subway scenario (Fig. 4). We see that even when global knowledge of the system is applied, and each node can estimate the percentage of peers that hold a given item, we still observe a considerable increase in the amount of public downloaded data. In fact, we obtain slightly better results with the weighted relay request strategy in terms of increased private goodput as well as decreased public goodput. The comparison with the optimal channel choice strategy is similar for the Östermalm scenario, but omitted due to space constraints (see [11]).

\section{B. Caching in low churn environments}

For our Train scenario we explore two different settings: Train (normal) where we keep the initial time schedule of the train but vary the mean number of passengers that enter the train at each station, and Train (extended) where we deliberately increase the trips between any two stations by a factor of 5. One can think of the Train (normal) scenario as a setting which represents the passenger dynamics on a particular line over different times of the day. The mean sojourn times for each of these scenarios is shown in Table II.

1) Train scenario with different arrivals: Fig. 5 presents the results for $\overline{G_{p r i}}$ and $\overline{G_{p u b}} / \overline{G_{p r i}}$ for the Train (normal) scenario with mean number of passengers $\lambda=20$ that enter the train at each station. Comparing these results to the results for the high churn scenarios, we see similar trends in the performance

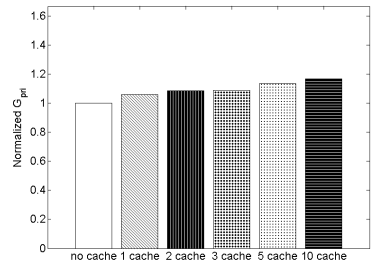

(a)

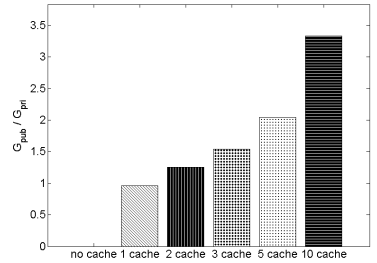

(b)
Fig. 4. (a) Normalized $\overline{G_{p r i}}$ and (b) $\overline{G_{p u b}} / \overline{G_{p r i}}$ with applied optimal channel choice strategy for the Subway scenario.

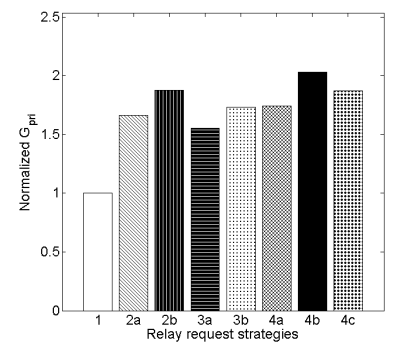

(a)

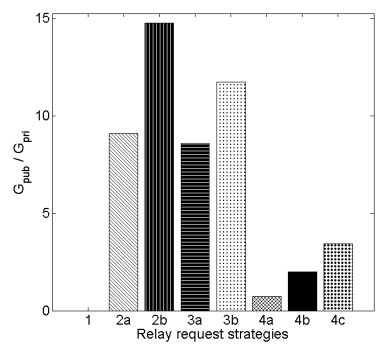

(b)
Fig. 5. (a) Normalized $\overline{G_{p r i}}$ and (b) $\overline{G_{p u b}} / \overline{G_{p r i}}$ with applied relay request strategy for the Train (normal) scenario with $\lambda=20$.

of the different relaying strategies. However, a more detailed investigation shows that the lower churn scenario enhances the performance of the weighted relay request strategy with $m=2$ allocated cache positions both in terms of increased private goodput, as well as decreased ratio of public to private goodput. Moreover, comparing the performance of strategies $3 \mathrm{~b}$ and $4 \mathrm{a}$, we can see that they achieve the same performance in terms of private goodput, but differ greatly in overhead from public downloads.

We further vary the average amount of passengers that enter the train at each station, and we explore two more scenarios: Train (normal) with $\lambda=10$ to represent parts of the day with low passenger intensity, and Train (normal) with $\lambda=40$ to illustrate rush hours. The results for $\overline{G_{p r i}}$ and $\overline{G_{p u b}} / \overline{G_{p r i}}$ are presented in Table III. Again, the weighted relay request with $m=2$ allocated positions gives best results in terms of the trade-off between private and public goodput, no matter the average amount of passengers per station. Note that the amount of private data obtained by nodes decreases as the number of nodes in the system increases (e.g. $\overline{G_{p r i}}$ is highest for $\lambda=10)$. Observe also the private goodput for the Train (normal) scenario with $\lambda=40$ : in this denser scenario the 3hop strategies provide lower private goodput than the 2-hop cases. One possible explanation for this is that the nodes do not get the chance to communicate with all their neighbors in an effective way, thus the virtual extension of the range becomes an obstacle rather than an advantage.

2) Train scenario with different travel times: We keep the average number of passengers entering the train to be $\lambda=20$; the results are presented in Fig. 6. Comparing the normalized 
TABLE III

NORMALIZED PRIVATE GOODPUT $\overline{G_{p r i}}$ AND RATIO $\overline{G_{p u b}} / \overline{G_{p r i}}$ FOR Train (normal) SCENARIOS

\begin{tabular}{|l|l|l|l||l|l|l|}
\hline Configuration & \multicolumn{3}{|c||}{ Private goodput $\overline{G_{\text {pri }}}$} & \multicolumn{3}{c|}{ Ratio $\overline{G_{p u b}} / \overline{G_{p r i}}$} \\
\hline \hline & Train & Train & Train & Train & Train & Train \\
& $\lambda=10$ & $\lambda=20$ & $\lambda=40$ & $\lambda=10$ & $\lambda=20$ & $\lambda=40$ \\
\hline No relaying & 1 & 1 & 1 & 0 & 0 & 0 \\
\hline Hop-limited relay, 2 hops & 1,90 & 1,66 & 0,86 & 9,94 & 9,09 & 10,8 \\
Hop-limited relay, 3 hops & 2,10 & 1,87 & 0,70 & 11,5 & 14,7 & 17,9 \\
\hline Greedy relay request with 2 hops limit & 1,77 & 1,55 & 1,24 & 6,67 & 8,60 & 7,43 \\
Greedy relay request with 3 hops limit & 2,39 & 1,73 & 1,08 & 10,1 & 11,7 & 9,85 \\
\hline Weighted relay request with $\mathrm{m}=2$ & 2,04 & 1,74 & 1,60 & 0,55 & 0,74 & 2,23 \\
Weighted relay request with $\mathrm{m}=4$ & 2,25 & 2,03 & 1,70 & 1,27 & 2,00 & 6,84 \\
Weighted relay request with $\mathrm{m}=6$ & 2,05 & 1,87 & 1,63 & 2,34 & 3,42 & 9,18 \\
\hline
\end{tabular}

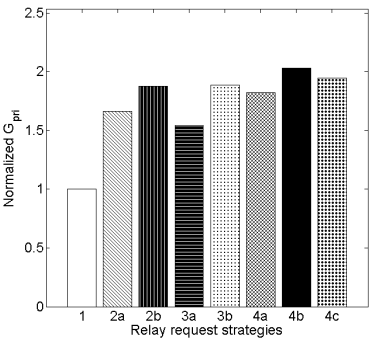

(a)

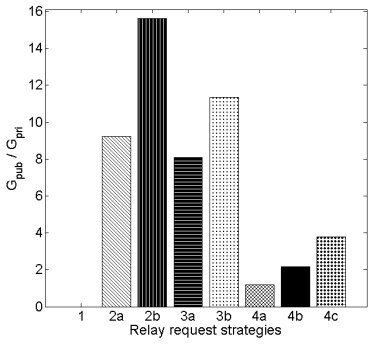

(b)
Fig. 6. (a) Normalized $\overline{G_{p r i}}$ and (b) $\overline{G_{p u b}} / \overline{G_{p r i}}$ with applied relay request strategy for the Train (extended) scenario with $\lambda=20$.

private goodput $\overline{G_{p r i}}$ for the Train (normal) and Train (extended) scenarios, Fig. 5(a) and 6(a), we see that the prolonged travel times do not affect drastically the amount of private data obtained by nodes in the train. The same observation holds for the ratio $\overline{G_{p u b}} / \overline{G_{p r i}}$ in Fig. 5(b) and 6(b). Although the longer times do provide slightly higher values in the public data cached in nodes, they are not significant and do not contribute to increasing private goodput. Thus, extended travel times lead to a steady-state in the system, and we reach a point when nodes have nothing more to exchange with their direct or indirect neighbors. We have to note, however, that this conclusion may not hold if nodes create and inject new content items in the system during their travels, or if subscriptions were for more than 10 out of 1000 items as used here.

\section{CONCLUSiON}

We have studied the usage of public caching for enhancing content dissemination in opportunistic networks in which data exchange is based purely on subscriptions. The main question we have addressed is whether nodes should fetch and store contents which are not of private interest. The study has been conducted with a complete system simulation. We have looked into two types of mobility: realistic pedestrian mobility to reflect two urban scenarios with high churn, and traincommuter mobility to represent low churn scenarios during different times of the day (e.g. early morning travels, and rush hour).

The simulation results suggest that when in high churn environments, public caching improves the application layer good- put. However, the vast majority of the proposed strategies have significantly increased overhead. We note that the proposed weighted relay request strategy with $m=2$ allocated positions for publicly requested content performs well, and significantly decreases the overhead from public content downloads while it still provides a relative improvement in private goodput. However, in dynamic settings with high churn, where nodes' sojourn times are relatively short, the effect of the weighted relay request strategy with $m=2$ diminishes. In such cases relying only on private caching can be sufficient.

The scenarios with low churn further enhance the performance of the weighted relay request strategy with $m=2$ allocated positions. However, we note that the effect of the weighted relay request, as well as the other proposed strategies, decreases as the node density in the scenario increases.

The optimal channel choice strategy from [5] does not perform better than our strategies.

\section{REFERENCES}

[1] O. R. Helgason, E. A. Yavuz, S. T. Kouyoumdjieva, L. Pajevic, and G. Karlsson, "A mobile peer-to-peer system for opportunistic contentcentric networking," in Proc. of ACM SIGCOMM MobiHeld, 2010.

[2] E. Yoneki, P. Hui, S. Chan, and J. Crowcroft, "A socio-aware overlay for publish/subscribe communication in delay tolerant networks," in Proc. of ACM MSWiM, 2007.

[3] C. Boldrini, M. Conti, and A. Passarella, "Contentplace: social-aware data dissemination in opportunistic networks," in Proc. of ACM MSWiM, 2008.

[4] Y. Ma, M. Kibria, and A. Jamalipour, "Cache-based content delivery in opportunistic mobile ad hoc networks," in IEEE GLOBECOM, 2008.

[5] L. Hu, J.-Y. Le Boudec, and M. Vojnovic, "Optimal channel choice for collaborative ad-hoc dissemination," in Proc. of IEEE INFOCOM, 2010.

[6] P. Costa, C. Mascolo, M. Musolesi, and G. Picco, "Socially-aware routing for publish-subscribe in delay-tolerant mobile ad hoc networks," IEEE Journal on Selected Areas in Communications, vol. 26, no. 5, pp. $748-760$, june 2008.

[7] P. Hui, J. Crowcroft, and E. Yoneki, "Bubble rap: social-based forwarding in delay tolerant networks," in Proc. of ACM MobiHoc, 2008.

[8] D. Gunawardena, T. Karagiannis, A. Proutiere, E. Santos-Neto, and M. Vojnovic, "Scoop: decentralized and opportunistic multicasting of information streams," in Proc. of MobiCom, 2011.

[9] O. Helgason, S. T. Kouyoumdjieva, and G. Karlsson, "Does mobility matter?" in Proc. of WONS, 2010.

[10] O. R. Helgason and K. V. Jónsson, "Opportunistic networking in omnet++," in Proc. of SIMUtools, 2008.

[11] S. Chupisanyarote, "Content caching in opportunistic wireless networks," Master's thesis, Royal Institute of Technology, KTH, 2011. 